\title{
FPGA Implementation of High Speed Digital FIR Filter
}

\author{
Lakshmi kiranMukkara \\ Department of Electronics \& Communication Engineering, Yogi Vemana University,Proddatur \\ Email: lk.ece@yogivemanauniversity.ac.in \\ Kota VenkataRamanaiah \\ Department of Electronics \& Communication Engineering, Yogi Vemana University,Proddatur \\ Email: ramanaiahkota@yogivemanauniversity.ac.in
}

\begin{abstract}
Multipliers play an important role in today's digital world especially in DSP and DIP applications. With advances in technology, many researchers are trying to design multipliers which offer either of the following targets-high speed, low power consumption, regularity of layout and less area or even combination of them in one multiplier. Now-a-days researchers are focusing on low power and high speed. This may be possible by designing effective multiplies as these are involved in huge amount in almost all DSP architectures. To do so many multipliers are available such as Booth multiplier and Wallace tree multiplier. Booth multiplier gives high performance (accuracy) but with high delay, whereas Wallace tree multiplier gives high speed but with less accuracy.The proposed method of CSD multiplication has the ability to reduce truncation error, computation latency for constant word length multiplications. Furthermore Horner CSD multiplier with pipelining concept makes multiplier design power efficient and speed efficient. This novel CSD algorithm is used here to design a Digital FIR filter and the same is designed with the booth and Wallace tree algorithm and compared. It is observed that proposed design is better w.r.t no. of slices and delay in comparison with existing methods. This design is coded in Verilog HDL and targeted on the spartran-3 device of XILINX 14.5.
\end{abstract}

Keywords -CSD (Canonic Signed Digit), DSP (Digital Signal Processing), DIP (Digital Image Processing), Verilog HDL (Hardware Description Language), VHDL (Very high speed HDL).

Date of Submission: June 15, 2018

Date of Acceptance: Aug 10, 2018

\section{INTRODUCTION}

The current digital world makes most works of human being just in few steps. Still delay is to be reduced along with power consumption. Delay can be reduced at various levels such as algorithmic level, architecture level, coding level, and physical level and etc. In this work architecture level is considered to design speed efficient multiplier. Here multiplier is considered on behalf of VLSI chip as it constitutes a basic component and that to contain in huge number.Popular architectures for multipliers available are Booth multiplier [10], Wallace tree multiplier [11] and CSD multiplier. Design of Simple FIR filter is considered here for illustration point of view. Booth multiplier suffers from delay, and Wallace tree multiplier suffers from accuracy. They can be overcomed with CSD multiplier.

Simple FIR filter along with Booth multiplication algorithm \& Wallace Tree multiplication algorithm is discussed in Section-II, CSD multiplication algorithm for FIR filter design is discussed in Section-III, Results of these filters along with their comparison is presented in Section-IV, and conclusion of this work is presented in the last Section-V.

\section{BOOTH MULTIPLIER AND WALLACE TREE MULTIPLIER FOR FIR FILTER DESIGN [10]}

Architecture of FIR Filter: Direct form of FIR filter is considered in this work. This FIR filter of order $\mathrm{M}$ with coefficients $M+1$ requires $M$ 2-input adders and $M+1$ multipliers. The architecture of direct form-2 FIR filter structure is as shown in Figure 1.

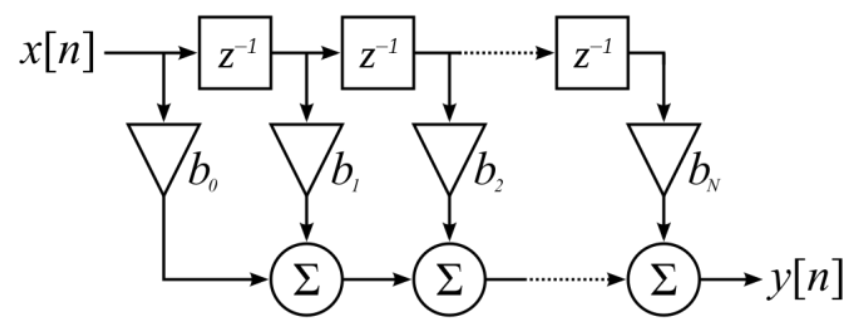

Figure 1: Direct form-2 FIR filter structure Input output relations of a FIR filter is given by

$$
y(n)=\sum_{k=0}^{M-1} h_{k} x(n-k) \Leftrightarrow H(z)=\sum_{k=0}^{M-1} h_{k} z^{-k}
$$

Where $\mathrm{x}$ is input coefficients and $\mathrm{h}$ is filter coefficients. Output of the filter requires both multiplier and adder. Efficient design of these units decides overall efficiency of 
FIR filter design. For this, Booth multiplier and Wallace Tree multiplier are considered in this section and CSD multiplier case is discussed in the next section.

Booth Algorithm: This is a multiplication algorithm to multiply signed binary numbers in 2's complementary form. Step by step procedure for Booth multiplication is illustrated in Figure 2, and Hardware implementation of it is as shown in Figure 3.

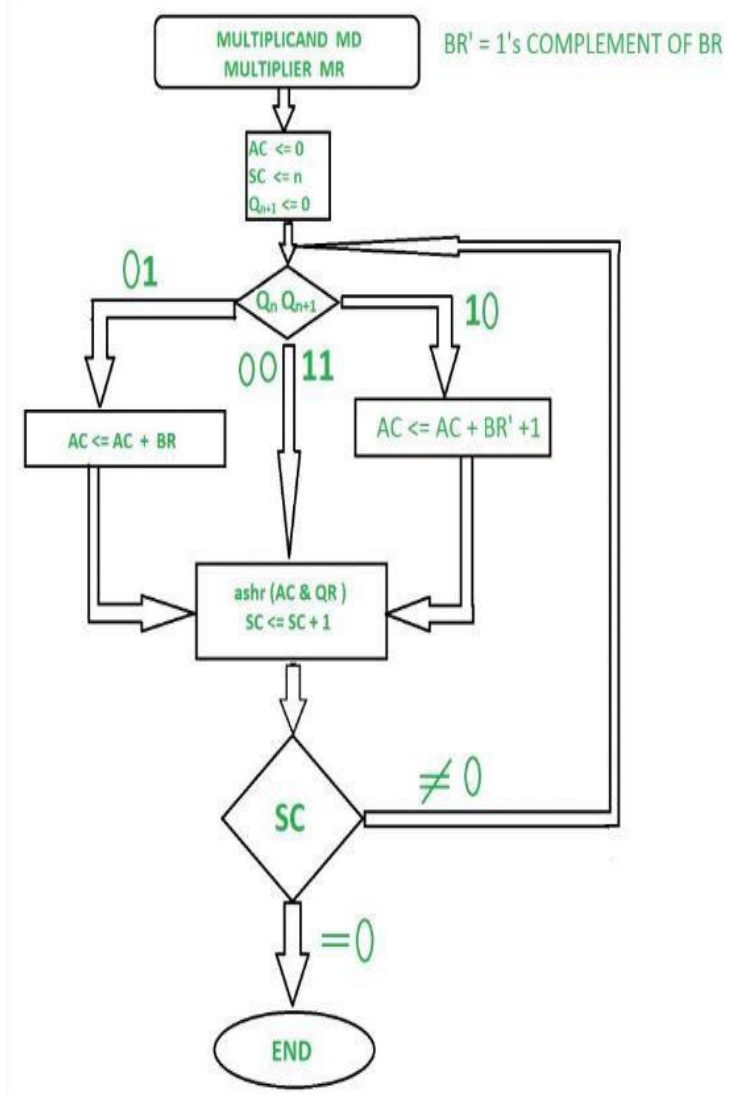

Figure 2: Flow chart for Booth Multiplication algorithm

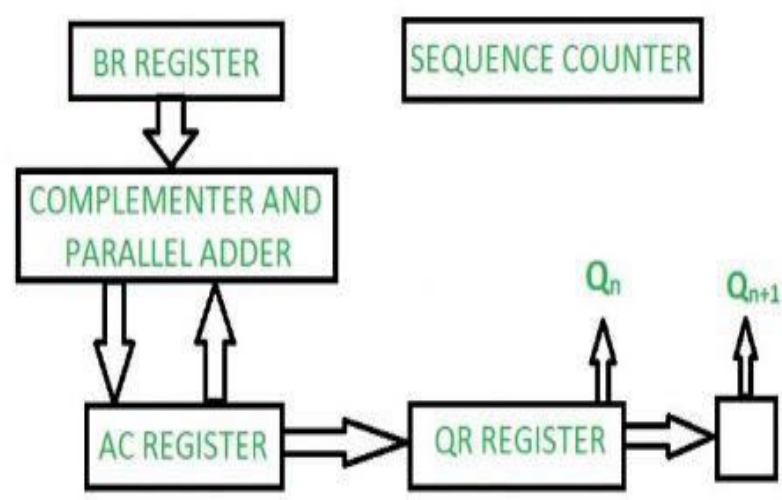

Figure 3: Hardware implementation of Booth multiplier

Wallace Multiplier: A Wallace tree is an efficient hardware implementation [11] of a digital circuit that multiplies two integers. It has 3 steps partial product generation stage, partial product reduction stage, and

partial product addition stage. These are illustrated in Figure 4.

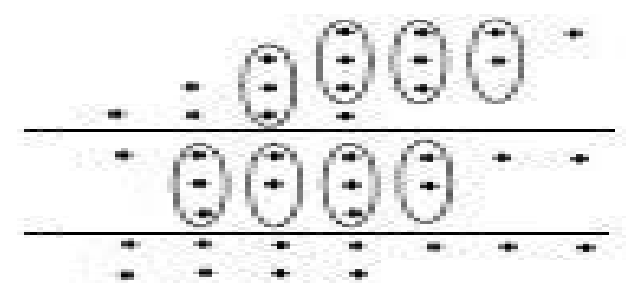

Figure 4: Wallace structure for a 4-bit multiplier

\section{CSD ALGORITHM FOR FIR FILTER DESIGN}

Although CSD algorithm seems to be similar to Booth algorithm, but CSD algorithm[1,2] results in $n / 3$ partial products instead of $\mathrm{n} / 2$ partial products in Booth algorithm. Hence power consumption will be further reduced with CSD algorithm. An algorithm for converting 2's complements binary number to CSD number is as shown in below and multiplication of converted CSD number with the normal binary number is as shown in Figure 5.

$$
\begin{aligned}
& \hat{a}_{-1}=0 \\
& \gamma-1=0 \\
& \hat{a}_{W}=\hat{a}_{W-1} \\
& \text { for }(i=0 \text { to } W-1) \\
& \{ \\
& \theta_{i}=\hat{a}_{i} \oplus \hat{a}_{i-1} \\
& \gamma_{i}=\overline{\gamma-1} \theta_{i} \\
& a_{i}=\left(1-2 \hat{a}_{i+1}\right) \gamma_{i} \\
& \}
\end{aligned}
$$

MULTIPLICAND

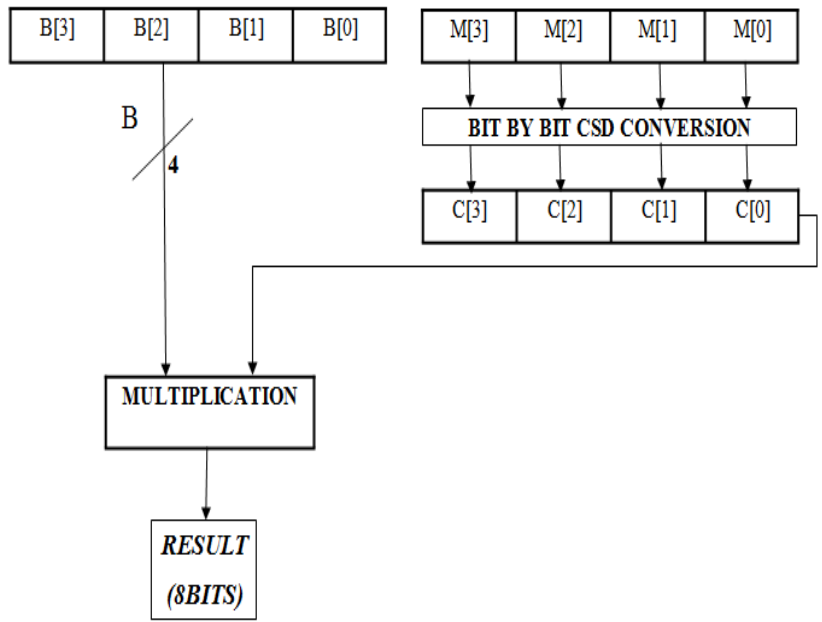

Figure 5: Pipelined CSD multiplication [3] 


\section{RESULTS \& COMPARISION}

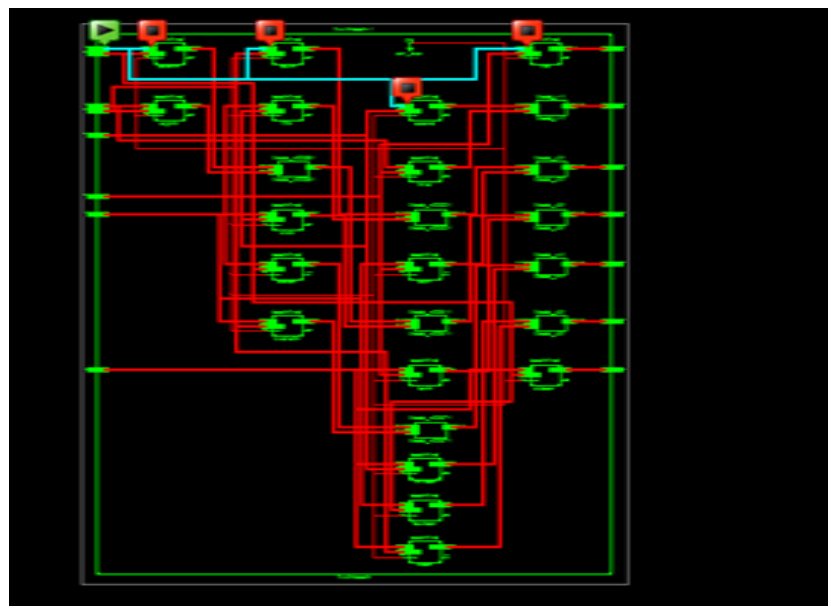

Figure 6:RTL Schematic of Booth multiplier based FIR filter

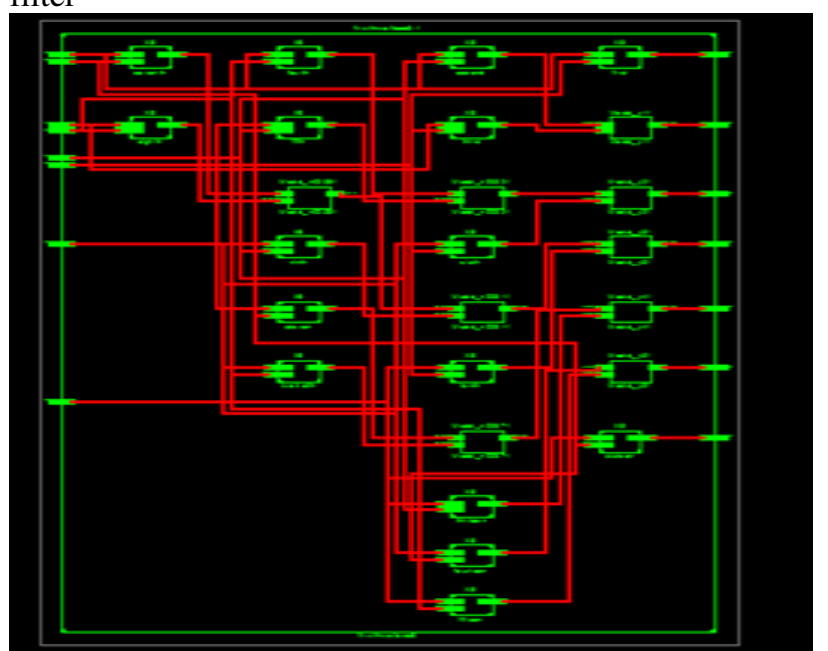

Figure 7: RTL Schematic of Wallace Tree multiplier based FIR filter

Table1: Comparision of FIR filter with different multipliers.

\begin{tabular}{|c|c|c|c|}
\hline Parameter & $\begin{array}{c}\text { FIR with } \\
\text { Booth } \\
\text { multiplication } \\
{[10]}\end{array}$ & $\begin{array}{c}\text { FIR with } \\
\text { Wallace tree } \\
\text { multiplication } \\
{[11]}\end{array}$ & $\begin{array}{l}\text { FIR with } \\
\text { CSD } \\
\text { multiplicati } \\
\text { on } \\
\end{array}$ \\
\hline $\begin{array}{l}\text { Number of } \\
\text { slice LUTs }\end{array}$ & $579 / 63400$ & $3492 / 63400$ & $42 / 63400$ \\
\hline $\begin{array}{c}\text { Number of } \\
\text { fully used } \\
\text { LUT- FF } \\
\text { pairs }\end{array}$ & $103 / 588$ & $224 / 3492$ & $0 / 42$ \\
\hline $\begin{array}{c}\text { Number of } \\
\text { bonded } \\
\text { IOBs }\end{array}$ & $89 / 210$ & $177 / 210$ & $56 / 210$ \\
\hline Delay & 31.426 & $10.962 \mathrm{~ns}$ & $3.627 \mathrm{~ns}$ \\
\hline Memory & $\begin{array}{c}313604 \\
\text { kilobytes }\end{array}$ & $\begin{array}{c}345796 \\
\text { kilobytes }\end{array}$ & $\begin{array}{c}\text { 312836kilo } \\
\text { bytes }\end{array}$ \\
\hline $\begin{array}{c}\text { Power } \\
\text { consumptio } \\
\mathrm{n}\end{array}$ & $42 \mathrm{mw}$ & $42 \mathrm{mw}$ & $42 \mathrm{mw}$ \\
\hline
\end{tabular}

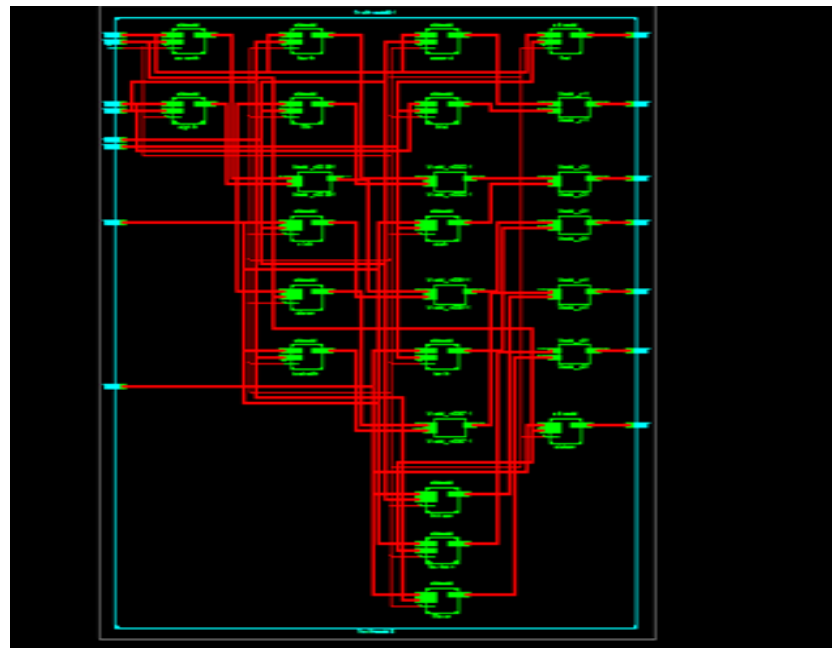

Figure 8: RTL Schematic of CSD based FIR filter

Figure $6,7 \& 8$ shows the RTL schematic of FIR filter based on Booth multiplier, Wallacetree multiplier and CSD multiplier and corresponding synthesis results are compared in Table.1.

Table2: Delay comparison

\begin{tabular}{|c|c|}
\hline Author & Delay \\
\hline Mitsuru Yamada [9] & $25.3 \mathrm{~ns}$ \\
\hline Abhijit Chandra [8] & $30 \mathrm{~ns}$ \\
\hline Proposed & $3.67 \mathrm{~ns}$ \\
\hline
\end{tabular}

In this work, a new efficient FIR filter can be designed using CSD.This reduces truncation errors and delay compared with other multipliers. i.e, existing techniques requires 10ns whereas proposed technique requires 3.672ns. This method using CSD format reduces the number of add operations during multiplication. CSD multiplication reduces number of slice LUTs by $92.74 \%$ in comparison with booth multiplication.CSD multiplication reduces number of slice LUTs by $98.79 \%$ in comparison with wallace tree multiplication.CSD multiplication reduces number of bonded IOBs by $37.07 \%$ in comparison with booth multiplication.CSD multiplication reduces number of bonded IOBs by $68.36 \%$ in comparison with wallace tree multiplication.CSD multiplication reduces delay by $66.91 \%$ in comparison with wallace tree multiplication and The method gives exact results with reduced number of add operations. Table. 2 shows the comparison of existed CSD multipliers with proposed CSD multiplier and it is shown that proposed CSD multiplier is very better in terms of delay it takes.

\section{Conclusion}

In this work, a new efficient FIR filter can be designed using CSD . This reduces truncation errors and delay compared with other multipliers. This method using CSD format reduces the number of add operations during multiplication. CSD multiplication reduces number of slice LUTs in comparison with previous multiplication 
techniques. CSD multiplication reduces delay in comparison with previouswallace tree and booth multiplication techniques. This method gives exact results with reduced number of add operations.In future there existsnew design,where designers can reduce the area occupied by the adder in various technologies. This work has been designed for 8-bit word size and results are evaluated for parameters like delay and power.This work can be further extended for higher number ofbits. New architectures can be designed in order to reduce the power, area and delay of the circuits. Steps may be taken to optimize the other parameters like frequency, number of gate clocks, length etc.

\section{REFERENCES}

[1] KeshabK.Parhi, vlsi digital signal processing systems: design and implementation, (John Wiley \&sons Publishing Company Inc., 1999).

[2] Shoab Ahmed Khan, multiplier-less multiplication by constants, in digital design of signal processing systems: a practical approach,( John Wiley \& sons Publishing Company,2010), pp.253-299.

[3] M.LakshmiKiran and K.VenkataRamanaiah, Implementation of High Speed and Low Area Digital Radix-2 CSD using Pipeline Concept, International Journal of Electronics and Communication Engineering, 10(1),2017, pp.53-61.

[4] Gustavo A.Ruiz, Mercedes Granda, efficient canonic signed digit recoding, Science Direct Microelectronics journal. [Online].42(2011) 10901097

[5] Yunhua Wan, Multiplier less CSD Techniques for high performance FPGA Implementation of Digital Filters, Ph.D. dissertation, Dept. School of Elect. And Computer Eng., University of Okalahoma., Norman, Okalahoma,2007.

[6] Gustavo A.Ruiz, Mercedes Granda, "efficient canonic signed digit recoding", ScienceDirect Microelectronics journal, Issue.42, pp.1090-1097, 2011.

[7] V.Sandhiya, Mr.S.Karthick², Dr.M.Valarmathy "A Survey of New Reconfigurable Architectures For Implementing FIR Filters With Low Complexity" International Conference on Computer Communication and Informatics (ICCCI -2014), Jan. $03-05,2014$, Coimbatore, INDIA

[8] Abhijit Chandra and SudiptaChattopadhyay "Differential Evolution based design of multiplier-less FIR filter using CSD Representation" IEEE Conference, 2012.

[9] Mitsuru Yamada and AkhinoriNishihaa, "High Speed FIR Digital Filter with CSD Coefficients Implemented on FPGA" Conference Paper on Jan 2001, DOI: 10.1145/370155.370201.

[10]D.Jaya Kumar, E.Logashanmugam,'Performance analysis of FIR filter using Booth multiplier",IEEE conference, July, 2014.

[11] Swati Chulet, Himanshu Joshi, "FIR Filter Designing Using Wallace Multiplier", International Journal of
Engineering and Technical Research (IJETR) ISSN: 2321-0869, Volume-3, Issue-6, June 2015.

\section{AUTHORS BIOGRAPHY}

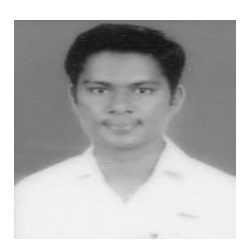

Mr.M.LakshmiKiran pursed B. Tech. from GPREC, Kurnool in 2009, M.Tech. from JNTUACEA, Ananthapuramu in 2014 with specialization in Digital Electronics and Communication Systems. $\mathrm{He}$ is currently Pursuing Ph.D (Full Time) on VLSI \& DIP from Yogi Vemana University, Proddatur since 2016. He has worked as Asst. Professor in Department of Electronics and Communication Engg. in VITS, Proddatur from 2014 to 2016. He has published 4 research papers in reputed international journals and conferences and it's also available online. His main research work focuses on VLSI, Digital Image Processing and Communication systems. He has 04 years of teaching experience.

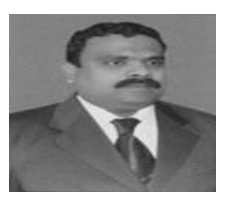

Dr.K.VenkataRamanaiah has obtained $\mathrm{Ph}$. D degree in the year 2009, from J.N.T.U College of Engineering Hyderabad in the Specialization of VLSI Architectures for Neural Network Based Image Compression, M. Tech degree from J.N.T.U College of Engineering, Hyderabad, in the Specialization of Digital Systems and Computer Electronics, and B.E Degree from KBNCE Gulbarga College, Gulbarga University. He is presently working as Head of the Department of Electronics and Communication Engineering, YSR Engineering College of Yogi Vemana University. Presently Guiding 7 Students for Ph.D. degrees. Professional Experience in teaching field is 24 Years. Areas of research interests are VLSI, Image processing, and Neural Networks. He has published more than 70 research papers in reputed international journals and conferences and it's also available online. 